\title{
BIOMARKERS OF MOUSE AGING
}

\section{Modifications of Minerals and Antioxidant Enzymes}

\author{
Heng-Kuan Wong, Jacqueline Riondel, and Alain Favier \\ Laboratoire de Biologie du Stress Oxydant (LBSO) \\ UFR de Pharmacie \\ Domaine de La Merci, 38700 La Tronche \\ France
}

Free radical damage has been reported to contribute to the etiology of many diseases and disorders including various types of cancer, autoimmune diseases, inflammatory dieseases, coronary heart diseases, photodermatoses, cataract, Alzheimer's disease, alcoholism.

There is evidence that free radical damage can contribute substantially to the etiology of the aging process.

To investigate the correlation between the oxidative stress and aging, we have determined the levels of lipid peroxidation in the brain, liver, kidneys, spleen and plasma from Swiss albino mice at ages of 4 and 14 months.

The results showed that the level of lipid peroxidation (measured as malondialdehyde) was significantly higher in older animals than in young animals.

The activities of erythrocyte antioxidant enzymes: glutathion peroxidase (GSH-Px $\mathrm{Se}$ ) and superoxide dismutase (SOD $\mathrm{Cu}-\mathrm{Zn}$ ) displayed an age-dependent elevation.

During aging process a significant increase of plasma Se level was observed but no significant alteration of plasma $\mathrm{Zn}$ level was noted.

Concerning the reduced glutathione (GSH) levels, no significant alteration was observed in most tissues except an age-dependent decline in the liver correlated with an oxidized glutathione (GSSG) level increase.

After extraction from liver the redox sensitive nuclear protein Sp1 showed an agedependent decline of its expression. NF. B considered as a stress oxidative inductible transcription factor displayed a significant increase of its expression and binding capacity during the aging process. 